\title{
Cancer-Associated Stroke: The Bergen NORSTROKE Study
}

\author{
Henriette Aurora Selvik ${ }^{a}$ b Lars Thomassen ${ }^{a}$, $\quad$ Anna Therese Bjerkreim ${ }^{a}$ b \\ Halvor Næss ${ }^{a-c}$ \\ a Department of Clinical Medicine, University of Bergen, and ${ }^{b}$ Department of Neurology, \\ Haukeland University Hospital, Bergen, and ${ }^{C}$ Centre for Age-Related Medicine, Stavanger \\ University Hospital, Stavanger, Norway
}

Key Words

Ischemic stroke · Cancer · Prothrombotic · Hypercoagulability · Stroke etiology

\begin{abstract}
Background: Underlying malignancy can cause ischemic stroke in some patients. Mechanisms include the affection of the coagulation cascade, tumor mucin secretion, infections and nonbacterial endocarditis. The release of necrotizing factor and interleukins may cause inflammation of the endothelial lining, creating a prothrombotic surface that triggers thromboembolic events, including stroke. The aims of this study were to assess the occurrence of cancer in patients who had recently suffered an ischemic stroke and to detect possible associations between stroke and cancer subtypes. Methods: All ischemic stroke patients registered in the Norwegian Stroke Research Registry (NORSTROKE) as part of the ongoing Bergen NORSTROKE study were included. Blood samples were obtained on admission. Stroke etiology was determined by the Trial of Org 10172 in Acute Stroke Treatment (TOAST) criteria, and the severity of stroke was defined according to the National Institute of Health Stroke Scale score. Information about cancer disease after stroke was obtained from patient medical records and The Cancer Registry of Norway. Results: From a total of 1,282 ischemic stroke patients with no history of cancer, 55 (4.3\%) patients were diagnosed with cancer after stroke. The median time from stroke onset to cancer diagnosis was 14.0 months (interquartile range 6.2-24.5). Twenty-three (41.8\%) patients were diagnosed with cancer within 1 year and 13 (23.6\%) within 6 months. The most common cancer type was lung cancer (19.0\%). By Cox regression analysis, cancer after stroke was associated with elevated D-dimer levels on admittance $(p<$
\end{abstract}

The study has used data from the Cancer Registry of Norway. 
$0.001)$, age ( $p=0.01)$ and smoking $(p=0.04)$. Conclusions: Cancer-associated stroke is rare, and routine investigation for cancer seems unwarranted in acute ischemic stroke. However, in stroke patients with elevated levels of blood coagulation factors, C-reactive protein, higher age and a history of smoking, underlying malignancy should be considered. Our study suggests that an unknown stroke etiology does not predict malignancy.

(C) 2015 The Author(s)

Published by S. Karger AG, Basel

\section{Introduction}

A link between cancer and stroke has long been recognized [1]. Some studies suggest that stroke, in some cases, is caused by underlying malignancy [2,3]. Mechanisms include the affection of the coagulation cascade, tumor mucin secretion, infections and nonbacterial endocarditis [4]. The release of necrotizing factor and interleukins may cause inflammation of the endothelial lining, creating a prothrombotic surface that triggers thromboembolic events, including stroke [5, 6]. Elevated D-dimer and fibrinogen levels can indicate a cancerinduced prothrombotic state [7, 8]. Elevated C-reactive protein (CRP) may represent cancermediated inflammation [9]. Cancer patients are at an increased risk of stroke, especially within 1 year of cancer proliferation [10]. Though rarely, ischemic stroke has been seen as the first sign of underlying malignancy $[7,10]$.

The aims of this community-based study were to assess the occurrence of cancer in patients who had recently suffered an ischemic stroke. We hypothesized that some of the patients had an underlying malignancy at stroke onset, which contributed to a cancer-associated stroke. We also aimed to detect possible associations between stroke and cancer subtypes.

\section{Methods}

\section{Study Design}

All patients admitted to the Department of Neurology, Haukeland University Hospital, Bergen, Norway, with confirmed ischemic stroke were prospectively included in the Norwegian Stroke Research Registry (NORSTROKE) as part of the Bergen NORSTROKE study.

Ischemic stroke was defined in accordance to the Baltimore-Washington Cooperative Young Study Criteria as neurologic deficit lasting $>24 \mathrm{~h}$ or transient ischemic attacks where CT or MRI showed infarctions related to the clinical findings [11]. The severity of the stroke was assessed by the National Institute of Health Stroke Scale (NIHSS) on admittance. Shortterm outcome was determined by the modified Rankin Scale (mRS) score 7 days after stroke onset (or on discharge if the patient was discharged earlier than after 7 days). On admission, vital parameters and blood analyses were obtained. Clinical characteristics were registered by a neurologist $<1$ day after admission. Investigations included electrocardiogram, Duplex and transthoracic echocardiography or transesophageal echocardiography. The Trial of Org 10172 in Acute Stroke Treatment (TOAST) criteria were used to determine the cause of stroke [12].

Stroke risk factors including hypertension, atrial fibrillation (AF), persistent foramen ovale, diabetes mellitus, angina pectoris, coronary artery disease and tobacco use were registered. Hypertension, angina pectoris, acute myocardial infarction, peripheral artery disease and chronic or paroxystic AF were considered present if diagnosed by a physician any time before stroke onset or during admission for stroke. 
Table 1. Patient characteristics in ISP with and without later cancer

\begin{tabular}{lccc}
\hline & $\begin{array}{c}\text { Cancer diagnosis after } \\
\text { index stroke }(\mathrm{n}=55)\end{array}$ & $\begin{array}{c}\text { ISP with no history of } \\
\text { cancer }(\mathrm{n}=1,227)\end{array}$ & $\mathrm{p}$ \\
\hline Male, $\mathrm{n}$ & 34 & 694 & 0.4 \\
Female, $\mathrm{n}$ & 21 & 533 & 0.1 \\
Mean age \pm SD, years & $72.7 \pm 11.5$ & $69.5 \pm 15.1$ & 0.9 \\
Median NIHSS (IQR) & $4(2-9)$ & $3(1-8)$ & 0.4 \\
Median mRS (IQR) & $2(1-4)$ & $2(1-4)$ & 0.9 \\
Mean temperature on admission $\pm \mathrm{SD},{ }^{\circ} \mathrm{C}$ & $36.6 \pm 0.8$ & $36.6 \pm 2.8$ & 0.04 \\
Prior ischemic stroke & $13(23.6)$ & $169(13.8)$ & 0.7 \\
Hypertension & $26(47.3)$ & $619(50.4)$ & 0.3 \\
Chronic AF & $6(10.9)$ & $88(7.2)$ & 0.5 \\
Paroxysmal AF & $6(10.9)$ & $107(8.7)$ & 0.3 \\
Diabetes mellitus & $10(18.2)$ & $170(13.9)$ & 0.6 \\
Previous myocardial infarction & $6(10.9)$ & $124(10.1)$ & 0.8 \\
Heart disease & $5(0.9)$ & $664(54.1)$ & 0.3 \\
Smoking & $36(65.5)$ & & 0.003 \\
\hline Blood values & & $1.4 \pm 2.4$ & $<0.001$ \\
Mean D-dimer \pm SD, mg/l & $2.7 \pm 4.6$ & $14.3 \pm 0.9$ & 0.2 \\
Mean fibrinogen \pm SD, mmol/l & $4.7 \pm 5.3$ & $6.7 \pm 2.5$ & 0.1 \\
Mean hemoglobin \pm SD, g/dl & $14.0 \pm 1.9$ & $10.0 \pm 26.4$ & 0.02 \\
Mean glucose \pm SD, mmol/l & $7.2 \pm 2.9$ & $5.4 \pm 1.3$ & 0.1 \\
Mean CRP \pm SD, mg/l & $18.8 \pm 41.2$ & & \\
Mean cholesterol \pm SD, mmol/l & $5.1 \pm 1.3$ & & \\
\hline
\end{tabular}

Values are expressed as n (\%), unless otherwise specified.

The study period started in February 2006 and ended on September 15, 2011. Study end points were September 15, 2011, or the date of death. Cancer data was obtained from the ischemic stroke patients (ISP) medical records and the Cancer Registry of Norway.

The study was approved by the Norwegian Regional Ethics Committee.

\section{Statistics}

The patients formed two groups; the ISP who were diagnosed with a new diagnosis of cancer after stroke onset (cancer-ISP) and the ISP who had no history of cancer (non-cancerISP) before or after stroke. Student's t test and $\chi^{2}$ analyses were used for the univariate analyses when appropriate. The Cox regression model was applied for multivariate analyses, and the statistical software Stata 13.0 was used for the statistical analyses.

\section{Results}

From a total of 1,282 patients with confirmed ischemic stroke, 55 (4.3\%) patients had one or two cancer diagnoses after their stroke. The mean age in the cancer-ISP group was 72.7 years [standard deviation (SD) 11.5], and for the non-cancer-ISP group it was 69.5 years (SD 15.1). The median follow-up time of the entire study population was 26.9 months [interquartile range (IQR) 13.7-42.5], while for non-cancer-ISP alone ( $n=1,227$ ), the median follow-up time was 27.7 months (IQR 14.2-43.1). Table 1 shows the baseline characteristics of all ISP. 

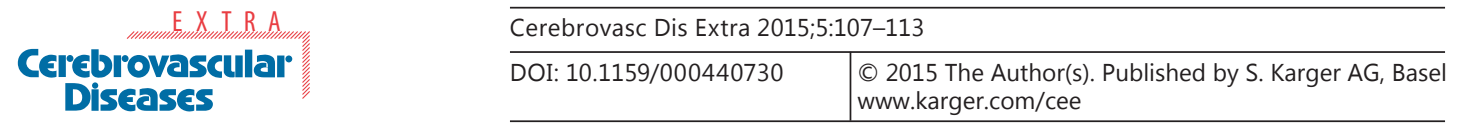

Selvik et al.: Cancer-Associated Stroke: The Bergen NORSTROKE Study

Table 2. Subtypes of cancer versus non-cancer ISP

\begin{tabular}{|c|c|c|c|c|c|c|c|c|}
\hline & $\begin{array}{l}\text { No cancer } \\
(\mathrm{n}=1,227)\end{array}$ & $\begin{array}{l}\text { Lung } \\
(n=12)\end{array}$ & $\begin{array}{l}\text { Prostate } \\
(n=10)\end{array}$ & $\begin{array}{l}\text { Colorectal } \\
(n=7)\end{array}$ & $\begin{array}{l}\text { Breast } \\
(n=7)\end{array}$ & $\begin{array}{l}\text { Gyn. cancer } \\
(n=5)\end{array}$ & $\begin{array}{l}\text { CUP } \\
(n=4)\end{array}$ & $\begin{array}{l}\text { Lymphoma } \\
(n=4)\end{array}$ \\
\hline Age & $69.5 \pm 15.1$ & $68.4 \pm 11.2$ & $75.2 \pm 12.4$ & $74.6 \pm 14.6$ & $76.5 \pm 8.9$ & $71.7 \pm 13.7$ & $62.4 \pm 5.4$ & $71.6 \pm 12.6$ \\
\hline Male, n (\%) & $694(56.6)$ & $6(50.0)$ & $10(100.0)$ & $5(71.4)$ & $1(14.3)$ & - & $4(100.0)$ & $3(75.0)$ \\
\hline Median NIHSS (IQR) & $3(1-8)$ & $5(1-8)$ & $4(3-9)$ & $3(0-9)$ & $2(2-3)$ & $5(4-7)$ & $3(2-7.5)$ & $7(4.5-10)$ \\
\hline Median mRS (IQR) & $2(1-4)$ & $2(1-4)$ & $1.5(1-3)$ & $2(2-4)$ & $2(2-3)$ & $1(1-1)$ & $2(81.5-2)$ & $2.5(1.5-3)$ \\
\hline \multicolumn{9}{|l|}{$\begin{array}{l}\text { Median time from } \\
\text { stroke to cancer }\end{array}$} \\
\hline (IQR), months & - & $8.7(5.5-27.3)$ & $14.0(6.0-20.3)$ & $4.0(1.3-22.0)$ & $12.2(8.1-25.8)$ & $14.3(2.2-18.1)$ & $16.8(7.1-23.8)$ & $15.9(12.4-20.1)$ \\
\hline Smoking, n (\%) & $664(54.1)$ & $10(83.3)^{*}$ & $7(70.0)$ & $5(71.4)$ & $3(42.9)$ & $3(60.0)$ & $4(100)^{* *}$ & $2(50.0)$ \\
\hline \multicolumn{9}{|l|}{ Blood values } \\
\hline Fibrinogen, mmol/l & $3.7 \pm 1.1$ & $4.0 \pm 1.5$ & $4.3 \pm 1.6$ & $9.2 \pm 13.6^{* *}$ & $4.5 \pm 2.1$ & $3.7 \pm 0.4$ & $3.6 \pm 0.4$ & $3.5 \pm 0.8$ \\
\hline Hemoglobin, g/dl & $14.3 \pm 1.5$ & $13.9 \pm 2.2$ & $14.0 \pm 1.9$ & $13.5 \pm 2.8$ & $13.2 \pm 2.0$ & $13.9 \pm 1.8$ & $14.8 \pm 1.3$ & $14.1 \pm 0.9$ \\
\hline Cholesterol, mmol/l & $5.3 \pm 1.3$ & $5.2 \pm 1.4$ & $5.0 \pm 1.0$ & $4.9 \pm 1.5$ & $5.0 \pm 1.7$ & $5.0 \pm 1.3$ & $5.5 \pm 0.4$ & $5.2 \pm 1.4$ \\
\hline D-dimer, mg/l & $1.4 \pm 2.5$ & $3.7 \pm 6.3^{*}$ & $1.7 \pm 1.4$ & $1.0 \pm 1.1$ & $1.8 \pm 2.7$ & $3.5 \pm 4.8$ & - & $3.0 \pm 1.8$ \\
\hline $\mathrm{CRP}, \mathrm{mg} / \mathrm{l}$ & $10.0 \pm 26.4$ & $29.7 \pm 68.4^{*}$ & $14.3 \pm 33.7$ & $12.3 \pm 21.3$ & $25.6 \pm 34.8$ & $31.6 \pm 59.5$ & $3.5 \pm 2.5$ & $3.0 \pm 1.8$ \\
\hline Glucose, mmol/l & $6.7 \pm 2.5$ & $8.2 \pm 4.6^{*}$ & $6.2 \pm 1.3$ & $6.7 \pm 1.5$ & $6.7 \pm 2.8$ & $6.5 \pm 1.8$ & $8.1 \pm 2.0$ & $6.2 \pm 1.8$ \\
\hline
\end{tabular}

Values are expressed as mean \pm SD, unless otherwise specified. ${ }^{*} \mathrm{p} \leq 0.05 ;{ }^{* *} \mathrm{p} \leq 0.01$.

Time from Index Stroke to Cancer Diagnosis

The median time from stroke onset to cancer diagnosis was 14.0 months (IQR 6.2-24.5). Twenty-three (41.8\%) patients were diagnosed within 1 year, $13(23.6 \%)$ within 6 months and $9(16.4 \%)$ within 3 months after stroke onset, including 4 (7.3\%) diagnosed within 1 month. Three were diagnosed within 1 week after stroke onset with the following cancer diagnoses: lung cancer, prostate cancer and lymphoma. Of the patients diagnosed with cancer within 1 month, 2 had been diagnosed with lung cancer. Colorectal cancer $(n=4)$ had the shortest median time from stroke onset to cancer diagnosis, namely 4.0 months (IQR 1.3-22.0), followed by lung cancer $(n=12)$ with a median time from stroke onset to cancer diagnosis of 8.7 months (IQR 5.5-27.3).

\section{Cancer Diagnoses}

In total, the 55 patients had 64 cancer diagnoses, distributed among 13 different cancer types. The 7 most common cancer types were lung cancer $(n=12,19.0 \%)$, prostate cancer $(n=10,15.9 \%)$, colorectal cancer $(n=7,11.1 \%)$, breast cancer $(n=7,11.1 \%)$, gynecological cancer $(n=5,7.9 \%)$, lymphoma $(n=4,6.3 \%)$ and metastatic cancer of unknown primary site (CUP; $n=4,6.3 \%$ ). Table 2 describes the characteristics of the cancer subtypes. The 6 other types of cancer included 4 bladder, 4 gastric and 3 pancreatic cancers, 2 carcinomas in situ, 1 malignant melanoma and 1 sarcoma.

\section{Smoking}

There was no difference in smoking habits between the cancer-ISP group and the non-cancer-ISP control group. However, by cancer subtypes, lung cancer $(p=0.03)$ and lymphoma $(p=0.003)$ had a higher rate of smoking compared to non-cancer-ISP.

\section{Blood Samples}

CRP was elevated in the cancer-ISP on admittance ( $\mathrm{p}=0.02)$. Among cancer subtypes, only lung cancer had increased mean CRP levels of 29.7 (SD 68.4, p $\leq 0.05$ ). The D-dimer level was elevated in the cancer-ISP on admittance $(p=0.003)$. Among the cancer subtypes, lung cancer patients had increased D-dimer levels compared to ISP with a mean D-dimer of 3.7 (SD $6.3, \mathrm{p} \leq 0.05$ ). 


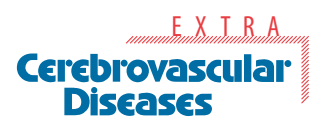

Table 3. Cox regression analysis in ISP with and without cancer after stroke

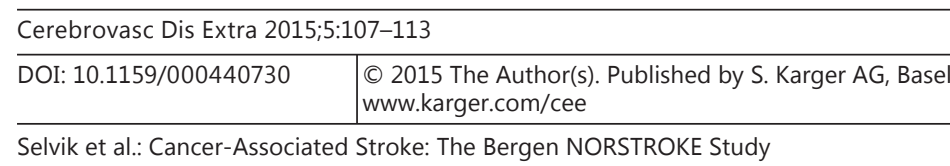

\section{Stroke Etiology and Outcome}

Of the 55 cancer-ISP, 21 (38.2\%) had an unknown stroke etiology compared to 516 $(42.1 \%)$ of the non-cancer-ISP ( $p=0.09$ ). Cardioembolic etiology was found in $32.7 \%$ of the cancer-ISP and $28.5 \%$ of the non-cancer-ISP. Among cancer subtypes, lymphoma had a higher frequency of cardioembolic etiology $(\mathrm{p} \leq 0.05)$. The mRS score did not differ between non-cancer-ISP and cancer subtypes.

By Cox regression analysis, cancer after stroke was associated with increased D-dimer levels on admittance $(p<0.001)$, age $(p=0.01)$ and smoking $(p=0.04$; table 3$)$.

\section{Discussion}

We found that malignancy was detected in few patients $(0.3 \%)$ within the first month after acute ischemic stroke. However, several previous studies have shown that ischemic stroke can be the first manifestation of cancer $[2,7,13,14]$. One study found an underlying malignancy in $0.4 \%$ of their ischemic stroke patients [7]. We found that of all ischemic stroke patients with no prior cancer, $1.8 \%$ were diagnosed with cancer within 1 year. It has previously been reported that mainly advanced cancers create a massive risk [3]. For the ischemic stroke patients diagnosed with cancer within 6 months (1.0\%), it is likely that they had an underlying malignancy at the time of stroke onset.

Knowing that specific tumor types may affect the vascular system in different ways, and that some cancers have similar risk factors as for stroke, analyses of subtypes of cancer are important [15]. Lung cancer, prostate cancer, colorectal cancer, breast cancer, gynecological cancer, CUP and lymphoma were the most common cancer types in our study. Prothrombotic states have previously been documented in cancer of the lung and colon as well as lymphomas. Lung cancer was overrepresented in our study, which also has been reported by similar studies of cancer-associated stroke [16]. Gynecological tumors are also reported to have a potential for being included in ischemic stroke pathogenesis [13]. Of the prostate cancer patients, 50\% had an unknown stroke etiology. However, as prostate cancer is rarely mentioned in relation to prothrombotic state, our findings may be due to prostate cancer being the most common male cancer type in general and thus incidental to the stroke [17]. Both lung and colon cancer have been linked to smoking [18]. Smoking is also a risk of ischemic stroke and may be a confounding factor in some cancer-associated strokes.

Of the classic risk factors for ischemic stroke, smoking was the only one associated with cancer. Higher D-dimer and CRP levels were associated with cancer, especially with lung cancer. In concordance, previous studies have also shown a higher risk for stroke in lung cancer patients [19]. Increased D-dimer and CRP levels may be caused by cancer development [8, 9].

The ISP with cancer were older than those in the control group. However, looking at the subtypes of cancer, the patients diagnosed with lung cancer and CUP were slightly younger than the control group. It has been shown that younger patients, below 49 years of age, often 
have different stroke etiologies than older stroke patients; prothrombotic state is more common and classic risk factors such as atherosclerosis are less common [20].

Stroke severity was similar between the two groups. A previous study defined stroke as being cancer related if stroke etiology was unknown and cancer was diagnosed within 6 months of stroke [10]. In contrast, we found no difference in etiology between our two groups. Thus, etiology may not be a predictor of cancer-associated stroke.

A limitation to our study was that the follow-up time differed among the study subjects. This may have caused a possible underestimation of cancer occurrence. On the other hand, patients diagnosed with cancer within 6 months after stroke onset may have been under careful medical surveillance due to their strokes, increasing the possibility of early cancer detection [16]. Because of the likelihood of stroke involvement in various cancers, it was necessary to study the subtypes of cancer separately. In doing so, each group consisted of a low number of study subjects. An advantage of the present study, as compared to previous studies, is the exclusion of patients with prior cancer [19]. This eliminates the question of whether the cancer treatment or the malignancy itself was the potentially involved factor in stroke pathogenesis.

\section{Conclusion}

Cancer-associated stroke is rare, and routine investigation for cancer seems unwarranted in acute ischemic stroke. However, in stroke patients with elevated levels of blood coagulation factors, CRP, higher age and a history of smoking, underlying malignancy should be considered. Our study suggests that an unknown stroke etiology does not predict malignancy.

\section{Acknowledgements}

H.A.S. received funding from The Research Council of Norway.

\section{References}

1 Grisold W, Oberndorfer S, Struhal W: Stroke and cancer: a review. Acta Neurol Scand 2009;119:1-16.

$\checkmark 2$ Giray S, Sarica FB, Arlier Z, Bal N: Recurrent ischemic stroke as an initial manifestation of a concealed pancreatic adenocarcinoma: Trousseau's syndrome. Chin Med J (Engl) 2011;124:637-640.

-3 Kwon MH: Stroke as the first manifestation of concealed cancer. J Neurol Sci 2007;258:80-83.

$\checkmark 4$ Gonzalez Quintela A, Candela MJ, Vidal C, Roman J, Aramburo P: Non-bacterial thrombotic endocarditis in cancer patients. Acta Cardiol 1991;46:1-9.

5 Graus F, Rogers LR, Posner JB: Cerebrovascular complications in patients with cancer. Medicine (Baltimore) $1985 ; 64: 16-35$.

6 Bick RL: Cancer-associated thrombosis. N Engl J Med 2003;349:109-111.

7 Taccone FS, Jeangette SM, Blecic SA: First-ever stroke as initial presentation of systemic cancer. J Stroke Cerebrovasc Dis 2008;17:169-174.

8 Kono T, Ohtsuki T, Hosomi N, Takeda I, Aoki S, Sueda Y, et al: Cancer-associated ischemic stroke is associated with elevated D-dimer and fibrin degradation product levels in acute ischemic stroke with advanced cancer. Geriatr Gerontol Int 2012;12:468-474.

- Hassan Aref SR: CRP evaluation in non-small cell lung cancer. Egypt J Chest Dis Tubercul 2014;63:717-722.

10 Nguyen T, DeAngelis LM: Stroke in cancer patients. Curr Neurol Neurosci Rep 2006;6:187-192.

11 Johnson CJ, Kittner SJ, McCarter RJ, Sloan MA, Stern BJ, Buchholz D, et al: Interrater reliability of an etiologic classification of ischemic stroke. Stroke 1995;26:46-51. 
12 Adams HP Jr, Bendixen BH, Kappelle LJ, Biller J, Love BB, Gordon DL, et al: Classification of subtype of acute ischemic stroke. Definitions for use in a multicenter clinical trial. Toast. Trial of Org 10172 in Acute Stroke Treatment. Stroke 1993;24:35-41.

-13 Borowski A, Ghodsizad A, Gams E: Stroke as a first manifestation of ovarian cancer. J Neurooncol 2005;71: 267-269.

14 Cornuz J, Bogousslavsky J, Schapira M, Regli F, Camenzind E: Ischemic stroke as the presenting manifestation of localized systemic cancer. Schweiz Arch Neurol Psychiatr 1988;139:5-11.

15 Amico L, Caplan LR, Thomas C: Cerebrovascular complications of mucinous cancers. Neurology 1989;39:522526.

16 Lindvig K, Moller H, Mosbech J, Jensen OM: The pattern of cancer in a large cohort of stroke patients. Int J Epidemiol 1990;19:498-504.

17 Andersen S, Richardsen E, Nordby Y, Ness N, Storkersen O, Al-Shibli K, et al: Disease-specific outcomes of radical prostatectomies in Northern Norway; a case for the impact of perineural infiltration and postoperative PSA-doubling time. BMC Urol 2014;14:49.

18 Hannan LM, Jacobs EJ, Thun MJ: The association between cigarette smoking and risk of colorectal cancer in a large prospective cohort from the United States. Cancer Epidemiol Biomarkers Prev 2009;18:3362-3367.

19 Selvik HA, Thomassen L, Logallo N, Naess H: Prior cancer in patients with ischemic stroke: the Bergen NORSTROKE study. J Stroke Cerebrovasc Dis 2014;23:919-925.

20 Naess H: Cerebral infarction in young adults (in Norwegian). Tidsskr Nor Laegeforen 2007;127:751-753. 